\title{
Cellulose nanowhisker modulated 3D hierarchical conductive structure of carbon black/natural rubber nanocomposites for liquid and strain sensing application
}

\author{
Xiaodong Wu', Canhui Lu', Yangyang Han', Zehang Zhou ${ }^{1}$, Guiping Yuan ${ }^{2 *}$ and Xinxing \\ Zhang $^{1 *}$ \\ ${ }^{1}$ State Key Laboratory of Polymer Materials Engineering, Polymer Research Institute of Sichuan \\ University, Chengdu 610065, China \\ ${ }^{2}$ Analytic and Testing Center of Sichuan University, Chengdu 610065, China
}

\begin{abstract}
Conductive polymer composites with "brittle" conductive structure (i.e. low percolation threshold) are widely recognized as potential sensing materials because they may exhibit a unique stimuli-responsive change of resistance. In this work, we prepared carbon black $(\mathrm{CB}) /$ natural rubber $(\mathrm{NR})$ nanocomposites with a unique 3D hierarchical conductive structure by incorporation of cellulose nanowhiskers via latex assembly technology. This unique conductive structure endowed the CB/NR nanocomposites with very low electrical conductivity percolation threshold (1.65 vol\%), large liquid sensing capacity (4427), fast
\end{abstract}

\footnotetext{
*Corresponding author.

E-mail address: ygp_04@163.com (G. Yuan),xxzwwh@scu.edu.cn (X Zhang).
}

Tel: +86-28-85460607 Fax: +86-28-85402465. 
response rate (168 s), and good reproducibility. The role of the hierarchical conductive structure on the liquid sensing behavior was investigated, and the mechanism was also discussed. The potential application of this material as strain sensors was also evaluated. This study offers a universal, simple, low-cost and scalable approach for manufacturing flexible multifunctional sensing materials by modulating the microstructure of the traditional $\mathrm{CB} / \mathrm{NR}$ composites using natural cellulose resources.

Key words: carbon black, natural rubber, 3D hierarchical conductive structure, liquid sensor, strain sensor.

\section{Introduction}

Conductive polymer composites (CPCs), with the merits of light weight, low cost and good processability $^{[1-3]}$, have been exploited as remarkable "smart" materials for their response to various external stimuli such as strain ${ }^{[4-5]}$, pressure $^{[6]}$, presence of volatile organic liquids ${ }^{[7]}$, or temperature shifts ${ }^{[8-9]}$. The destruction and restruction of the percolated conductive networks in polymer matrix give electrical signal output, making it possible to detect environmental changes. However, not all CPCs are suitable to serve as sensing materials. As desirable sensing materials, a required electrical conductivity, a high response intensity, fast response, and good reproducibility are necessary for CPCs. Preparation of CPC sensing materials with these properties is still challenging. One of the critical issues is to fabricate continuous conductive network structures in CPCs with good balance of electrical conductivity and stimuli-sensitivity. Particularly, a well-constructed conductive network is necessary for high electrical conductivity and thus stable output signal, but perfect conductive networks are less sensitive to external stimuli, giving rise to a very weak output signal. Therefore, how to fabricate a "brittle" but effective conductive network structure in CPCs (i.e. low conductive fillers loading and satisfying conductivity) becomes the key issue for the construction of desirable sensing materials. 
Very recently, water-based latex assembly technique has been proved to be very effective in fabricating CPCs with low percolation thresholds from nanoscale conductive fillers ${ }^{[10-20]}$. Specifically, polymer latex is mixed uniformly with well dispersed conductive fillers. During the subsequent cocoagulation or drying process, conductive fillers are selectively located in the interstitial space between the polymer latex microspheres and assembled into a continuous 3D hierarchical network. CPCs with this structure exhibit lower percolation threshold and higher electrical conductivity compared to those with randomly dispersed fillers. However, almost all the conductive fillers for latex assembly technique focused on the expensive carbon nanotube (CNT) and reduced graphene oxide (rGO). The complex production process and high cost of CNT and rGO greatly hinder their large scale application.

Recently, we fabricated a unique 3D hierarchical conductive structure in natural rubber (NR) matrix using cheap and abundant carbon black $(\mathrm{CB})$ assisted by cellulose nanowhiskers $(\mathrm{CNs})^{[21]}$. The resultant composites showed much lower electrical conductivity percolation threshold (2.9 vol\%) compared to traditionally prepared $\mathrm{CB} / \mathrm{NR}$ composites. In our subsequent work, we interestingly found that the CNs modulated CB/NR composites without vulcanization exhibited further lower percolation threshold (1.65 vol\%), which is infrequent for CB based CPCs. CPCs with such a low percolation threshold (i.e. brittle conductive networks) is quite suitable to serve as potential sensing materials. However, no investigation, to the best of our knowledge, has been conducted to study the sensing performance of CPCs with CB based 3D hierarchical structure fabricated by latex assembly technique.

In this work, for the first time, we explored the sensing behaviors of the $\mathrm{CB} @ \mathrm{CNs} / \mathrm{NR}$ nanocomposites with this 3D hierarchical conductive structure. Interestingly, the resultant flexible $\mathrm{CB} @ \mathrm{CNs} / \mathrm{NR}$ nanocomposites exhibited desirable electrical conductivity, high but tunable liquid sensing capacity, and good reproducibility when exposed to organic liquids. Furthermore, this CB based CPC showed potential application as strain sensors. The desirable sensing capabilities of our 
$\mathrm{CB} @ \mathrm{CNs} / \mathrm{NR}$ nanocomposites, which are comparable with the recently reported sensing materials, together with their significant advantages of low cost, easy fabrication and environmental friendliness, make them good candidates for liquid and strain sensing materials .

\section{Materials and methods}

\subsection{Materials}

Analytical grade sulfuric acid $\left(\mathrm{H}_{2} \mathrm{SO}_{4}, 95-98 \mathrm{wt} \%\right)$, ammonium hydroxide $\left(\mathrm{NH}_{3} \cdot \mathrm{H}_{2} \mathrm{O}, 25-28 \%\right)$, toluene ( $\geqq 99.5 \%)$, chloroform (99.0 wt\%), petroleum ether, cyclohexane (99.5 wt\%), n-hexane (97.0 wt\%) and xylene (99.0 wt\%) were all purchased from Chengdu Kelong Chemical Reagent Company (China) and used without further purification. Carbon black (BP 2000) with particle size $15 \mathrm{~nm}$, nitrogen absorption special surface area $1500 \mathrm{~m}^{2} / \mathrm{g}$, and dibutyl phthalate absorption $330 \mathrm{~mL} / 100 \mathrm{~g}$ was obtained from Cabot Chem., USA. The NR latex (solid content: $58 \mathrm{wt} \%$ ) was provided by Chengdu Xinyuanding Co., Ltd (China). Medical purified cotton was obtained from Xuzhou Health Factory Co., Ltd (China).

\subsection{Preparation of CB@CNs nanohybrid}

CNs was prepared by controlled acid hydrolysis of cotton fibers according to our previous studies $^{[21]}$. For the preparation of CB@CNs nanohybrid, $43.32 \mathrm{~g}$ CNs suspension was diluted to 800 $\mathrm{mL}$ and sonicated for $5 \mathrm{~min}(1200 \mathrm{~W})$ to disperse the aggregation. After the $\mathrm{pH}$ value was adjusted to about 8.0 with $1 \mathrm{M} \mathrm{NH}_{3} \cdot \mathrm{H}_{2} \mathrm{O}, 0.2448 \mathrm{~g} \mathrm{CB}$ was added into the $\mathrm{CNs}$ suspension and the mixture was sonicated for $10 \mathrm{~min}(1200 \mathrm{~W})$, yielding well dispersed and suspended CB@CNs nanohybrid suspension.

\subsection{Preparation of $C B @ C N s / N R$ composites}

In a typical experiment, $3.44 \mathrm{~g}$ NR latex was diluted to $100 \mathrm{~mL}$ and stirred for a while. Then a desired amount of $\mathrm{CB} @ \mathrm{CNs}$ nanohybrids suspension and the NR latex were mixed uniformly, forming 
a black mixture of $\mathrm{CB} @ \mathrm{CNs}$ and NR latex. The final mixture was coagulated by adding $\mathrm{H}_{2} \mathrm{SO}_{4}(1 \mathrm{M})$. After filtering, the solid mixture was soaked in renewed distilled water to remove the residual $\mathrm{H}_{2} \mathrm{SO}_{4}$ and finally dried at $60{ }^{\circ} \mathrm{C}$ for $12 \mathrm{~h}$. The dried composites (about $0.7 \mathrm{~mm}$ ) were cut into pieces (30 $\mathrm{mm} \times 5 \mathrm{~mm}$ ) for liquid sensing tests. For strain sensing tests, $\mathrm{CB} @ \mathrm{CNs} / \mathrm{NR}$ composite was prepared following the same procedure of the above $\mathrm{CB} @ \mathrm{CNs} / \mathrm{NR}$ except coagulation by formic acid and casting into films $(0.12 \mathrm{~mm})$. For comparison, $\mathrm{CB} / \mathrm{NR}$ nanocomposites were prepared by direct tworoll mixing of $\mathrm{CB}$ and $\mathrm{NR}$.

\subsection{Characterization}

Transmission electron microscopy (TEM) was performed using a transmission electron microscope (JEOL JEM-100CX, Japan). Diluted CNs and CB@CNs nanohybrids aqueous suspensions were sonicated for $30 \mathrm{~min}$ to disperse the aggregation. The well dispersed $\mathrm{CNs}$ and $\mathrm{CB} @ \mathrm{CNs}$ nanohybrids suspensions were directly dropped on a copper grid for observation. The diameter of pristine CNs was measured using Image-J software and at least 100 particles from different TEM images were analyzed.

Preparation of drop-cast samples of $\mathrm{CB} @ \mathrm{CNs} / \mathrm{NR}$ nanocomposite for TEM observation: CB@CNs suspensions (containing $0.05 \mathrm{~g}$ CB@CNs nanohybrid) and $0.574 \mathrm{~g}$ NR latex (58 wt\%) were mixed and sonicated adequately for uniform mixing. The mixture was put on the copper grids and the water was evaporated. The final sample was observed using a transmission electron microscope (JEOL JEM-100CX, Japan).

$\mathrm{CB} @ \mathrm{CNs} / \mathrm{NR}$ nanocomposite (CB content: 5 vol\%) and CB/NR composites (CB content: 5 vol\%) prepared by conventional two-roll mixing method was cryo-microtomed using a Leica EM UC6 equipment to get the ultrathin cryo-sections of 70-80 nm thickness, which were collected and directly supported on a copper grid for observation. 
The electric conductivity of all samples were measured by a two-point measurement with a resistance meter (UT61, Uni-Trend, China) for $\mathrm{R} \leq 2 \times 10^{8} \Omega$ or using ZC36 high resistance instrument for $\mathrm{R}>2 \times 10^{8} \Omega$. A rectangle strip samples $(30 \mathrm{~mm} \times 5 \mathrm{~mm} \times 0.7 \mathrm{~mm})$ or square samples $\left(\Phi_{\mathrm{d}}=60 \mathrm{~mm}\right)$ were used for electrical measurement. The measured volume resistance $R_{V}(\Omega)$ was converted to volume resistivity $\rho_{\mathrm{v}}$ according to ASTM D4496 and D257 using the formula

$$
\rho_{\mathrm{v}}=\mathrm{R}_{\mathrm{v}} \frac{\mathrm{A}}{\mathrm{t}}
$$

Where $\mathrm{A}$ is effective area of the measuring electrode $\left(\mathrm{m}^{2}\right)$ and $\mathrm{t}$ is specimen thickness $(\mathrm{m})$.

For liquid sensing analysis, the $\mathrm{CB} @ \mathrm{CNs} / \mathrm{NR}$ composites $(30 \mathrm{~mm} \times 5 \mathrm{~mm} \times 0.7 \mathrm{~mm})$ at different $\mathrm{CB}$ loadings were used. The initial resistances of CB@CNs/NR nanocomposites filled with 2.5 vol\%, 3.75 vol\%, and 5.0 vol\% $\mathrm{CB}$ were $5.7 \times 10^{3}, 5.0 \times 10^{1}$, and $5.5 \mathrm{k} \Omega$, respectively. Liquid sensing tests were performed by quickly immersing the samples into a organic liquid at room temperature, and the resistance was real time recorded using a UT61 multimeter (measurement range: $1 \times 10^{-4} \Omega$ to $2 \times 10^{8} \Omega$ ). The electrodes were connected to both ends of the test samples using highly conductive metal clamps. For the drying step, the test sample was lifted up (remaining solvent drops were wiped off) and dried in hot-blast air, the resistance was also real time recorded. Different numbers of immersing-drying cycles were performed in order to check reversibility and reproducibility of the sample resistivity.

Before strain sensing test, the $\mathrm{CB} @ \mathrm{CNs} / \mathrm{NR}$ composite was pre-stretched until failure at a rate of $10 \mathrm{~mm} / \mathrm{min}$ to eliminate creep. Then the pre-stretched CB@CNs/NR strip $(80 \mathrm{~mm} \times 5 \mathrm{~mm} \times 0.05 \mathrm{~mm})$ was fixed on a versatile testing machine (ASTM D412-80). The electrodes of UT61 multimeter were connected to two points $(30 \mathrm{~mm})$ of the strips using highly conductive metal clamps. To test strain sensing, samples were subjected to ten loading-unloading cycles to $20 \%$ strain at a strain rate of $40 \%$ strain/mim. The resistance was real time recorded.

\section{Results and discussion}


The essence of our designed strategy for fabricating this CNs modulated multifunctional sensing material is schematically illustrated in Figure 1. CNs is derived by controlled acid hydrolysis of cotton fibers. When $\mathrm{CB}$ is added into $\mathrm{CNs}$ suspension under ultrasound, $\mathrm{CB}$ aggregates break up into individual $\mathrm{CB}$ nanoparticles and decorated on the surface of CNs, thus forming $\mathrm{CB} @ \mathrm{CNs}$ nanohybrid with good suspension stability and high aspect ratio. Then the $\mathrm{CB} @ \mathrm{CNs}$ nanohybrid suspension is uniformly mixed with NR latex. During the subsequent co-coagulation process, the NR latex microspheres create an excluded volume and essentially push the $\mathrm{CB} @ \mathrm{CNs}$ nanohybrid into the interstitial space between them, forming a continuous 3D hierarchical conductive structure. In drying process, water in interstitial space of NR latex microspheres is removed and NR latex microspheres are welded into bulk because of its self-adhesive nature. Thus, a CB based "brittle" but effective conductive network structure is fabricated in NR matrix. This CNs modulated CPC sensing material is expected to exhibit good response to various external stimuli.

We confirmed the feasibility of this CNs modulated fabrication of CB based 3D hierarchical conductive structure in NR as illustrated in Figure 1. Firstly, we observed the structure of pure CB using an optical microscope. Large-scale aggregates of $\mathrm{CB}$ with hundreds of micrometers were observed (Figure S1a), which could be visible to naked eyes clearly (inset picture). The large size of CB aggregates, which are much larger than NR microspheres (Figure S2), make it infeasible to fabricate conductive networks by latex assembly technique. While, after the hybridization with CNs by simple sonication, a uniform black CB@CNs nanohybrid suspension was obtained (Figure S1b). In addition, a red light pillar can be observed under a laser pointer (known as Tyndall effect), indicating that the CB@CNs nanohybrid suspension exhibited characteristics of colloid. These results reveal that CNs could serve as a stabilizer and enhance the dispersity and suspension stability of CB. 
Next, CB@CNs nanohybrid, as well as pristine CNs for comparison, was observed by TEM. As shown in Figure 2a, CNs were needle-like with the length of 200-500 nm and the width of 10-20 nm. After hybridization of $\mathrm{CB}$ and $\mathrm{CNs}$, individual $\mathrm{CB}$ particles are well deposited on the surface of CNs, forming a continuous nodular nano-sheath structure (Figure $2 \mathrm{~b}$ ). These results demonstrate that $\mathrm{CNs}$ acted as a good template for CB and could direct the arrangement of CB particles along CNs, yielding nodular CB@CNs nanohybrid with high aspect ratio, which makes it easier for CB@CNs nanohybrid to connect with each other and form continuous conductive networks.

The microstructure of CB@CNs nanohybrid and NR latex mixture was observed. As given in Figure 2c, CB@CNs nanohybrid is selectively located in the interstitial space between the NR latex microspheres and organized into a continuous network. This demonstrates that CNs could induce the selective location of $\mathrm{CB}$ to form a well modulated conductive structure in NR matrix by latex assembly technique. After co-coagulation and drying process, the CNs modulated 3D hierarchical conductive structure was maintained in the eventual CB@CNs/NR nanocomposite (Figure 2d).

To intuitively evaluate the 3D hierarchical conductive structure fabricated in NR matrix, the $\mathrm{CB} @ \mathrm{CNs} / \mathrm{NR}$ composite (5 vol\% CB) was etched with toluene to remove the NR matrix and the residual CB@CNs nanohybrid skeleton is characterized by SEM. As is shown in Figure 2e, a uniformly porous network of CB@CNs is clearly seen. The pore diameter varies from several hundred nanometers to several micrometers, which results from the extraction of one or several NR latex microspheres.

Importantly, this resultant $\mathrm{CB} @ \mathrm{CNs} / \mathrm{NR}$ nanocomposite exhibited excellent flexibility as shown in Figure $2 \mathrm{f}$, which makes it more attractive in various sensing fields. 
In general, microstructure of CPCs has significant influence on their properties. As shown in Figure 2g, significant electrical conductivity enhancement is observed for all CB@CNs/NR samples compared to $\mathrm{CB} / \mathrm{NR}$ composites. For example, the electrical conductivity of $\mathrm{CB} / \mathrm{NR}$ composite containing 3.75 vol\% CB is $4.8 \times 10^{-13} \mathrm{~S} / \mathrm{m}$, while for $\mathrm{CB} @ \mathrm{CNs} / \mathrm{NR}$ nanocomposites it reaches $3.5 \times 10^{-1}$ $\mathrm{S} / \mathrm{m}$ at the same loading fraction of $\mathrm{CB}$, showing a significant enhancement of about 12 orders of magnitude. Besides, a very low percolation threshold (ca. 1.65 vol\%) was realized for CB@CNs/NR composites (Figure 2h). The enhanced conductivity can be attributed to the 3D hierarchical conductive structure fabricated in NR, based on the stabilizing and template effect of CNs.

The liquid sensing properties of the CB@CNs/NR nanocomposites were determined by exposing them to organic solvents. Initially, we chose toluene, which is a good solvent for the NR matrix, as an typical analyte to evaluate the solvent sensing behaviors of $\mathrm{CB} @ \mathrm{CNs} / \mathrm{NR}$ nanocomposites. In order to obtain a visual understanding of the liquid sensing behavior, CB @CNs/NR nanocomposite with 5 vol\% CB, which could light up LED devices (Figure $2 \mathrm{~h}$ inset), was connected with a blue LED light. Thus, the electrical conductivity variation can be monitored by the illumination changes. As shown in Figure 3, during the immersing into toluene, the illumination faded over time and died out after 12 min, indicating the decreasing conductivity. Sample swelling could be observed clearly in this process. Then the sample was taken out and solvent drops remaining on the sample were wiped off using a tissue. During the drying process in hot-air, the blue LED light was lighted up again and the illumination intensified over time, along with sample shrinking. These intuitive illumination changes give a direct proof for the liquid sensing capacity of this CNs modulated CB/NR nanocomposites.

Desirable CPC sensing materials should balance the electrical conductivity and stimuli-sensitivity for strong and stable output signal. Hence, fabrication of an effective and sensitive conductive network structure in polymer matrix is necessary. With this aim in mind, we investigated the role of the CNs 
modulated hierarchical conductive structure on the liquid sensing properties by tuning CB@CNs loading. The resistance changes in test were converted into responsivity values, i.e. $R_{t} / R_{0}\left(R_{0}\right.$ is the initial resistance of the sample and $R_{t}$ is the measured resistance at time $t$ ).

As shown in Figure $4 \mathrm{a}, \mathrm{b}$ and $\mathrm{c}$, a gradual increase of resistance and then a drastic resistance change are observed for all samples during the immersing process, as a result of swelling of the NR matrix and breakage of the conductive networks. For $\mathrm{CB} @ \mathrm{CNs} / \mathrm{NR}$ with 2.5 vol\% $\mathrm{CB}$, the responsivity value (i.e. resistance variation) was 38.6 at the immersing time of 85 s. While for CB@CNs/NR with 3.75 vol\% $\mathrm{CB}$, the responsivity reached to 4427 at a longer immersing time of $168 \mathrm{~s}$. When CB loading of $\mathrm{CB} @ \mathrm{CNs} / \mathrm{NR}$ increased to 5.0 vol\%, the responsivity reached to 37743 at the immersing time of 560 s. These results can be ascribed to that higher conductive filler loading leads to better conductive networks, resulting higher responsivity but longer response time.

In the drying process, the resistance returned to the original levels for both $\mathrm{CB} @ \mathrm{CNs} / \mathrm{NR}$ nanocomposites with 3.75 vol\% and 5.0 vol\% CB because of the removal of organic liquid in NR and reconstruction of the conductive networks. It was noticed that the restoring time of resistance for $\mathrm{CB} @ \mathrm{CNs} / \mathrm{NR}$ filled with 3.75 vol\% CB is obviously longer than that of $\mathrm{CB} @ \mathrm{CNs} / \mathrm{NR}$ filled with 5.0 vol\% CB. This might be due to that it is more difficult for the junctions of conductive fillers to reconstruct in sparse conductive network fabricated with less conductive fillers. However, for $\mathrm{CB} @ \mathrm{CNs} / \mathrm{NR}$ filled with $2.5 \mathrm{vol} \% \mathrm{CB}$, the resistance showed just a slight decrease, but could not be restored to the original level. This indicates that conductive structure fabricated with low $\mathrm{CB}$ content more near the electrical percolation threshold tends to show irreversible breakage-reconstruction cycles. These results reveal that the liquid sensing performance can be tuned by fabricating various 3D hierarchical conductive networks using different CB@CNs loading. 
Plots of $\log \left(R_{t} / R_{0}\right)$ vs. time during the initial stage of immersing process (i.e. values of $R_{t} / R_{0}$ were in the range of $1 \sim 10$ ) were employed to evaluate the liquid sensing sensitivity of this 3D hierarchical conductive network structure to organic liquid stimuli. As mentioned above, $\log \left(\mathrm{R}_{\mathrm{t}} / \mathrm{R}_{0}\right)$ vs. time could reveal the rate of resistance variation. Higher rate of resistance variation indicates faster response of the sensing materials to stimuli ${ }^{[22]}$. As given in Figure 4 a', b' and c', linear slope of CB@CNs/NR nanocomposites filled with $2.5 \mathrm{vol} \%, 3.75 \mathrm{vol} \%$, and $5.0 \mathrm{vol} \% \mathrm{CB}$ were $0.0104,0.0072$, and 0.0039 , respectively. Higher linear slope signifies faster response ${ }^{[23]}$. Thus, conductive structure fabricated with low filler content is more sensitive to organic liquid stimuli. As desirable sensing materials, a required electrical conductivity, a high responsivity, and fast response are necessary for CPCs to obtain stable output signal and high sensitivity ${ }^{[24-25]}$. Hence, to balance the electrical conductivity and liquid sensing sensitivity, CB@CNs/NR nanocomposite filled with 3.75 vol\% CB is most suitable for organic liquid sensing in this work, which simultaneously possess much faster response (168 s) and higher responsivity (4427) when compared to other reported liquid sensing materials in literatures ${ }^{\text {[26-33] }}$ as shown in Table 1 . It is worth noting that $\mathrm{CB} / \mathrm{NR}$ composites prepared via traditional two-roll mixing method with same $\mathrm{CB}$ loadings failed to give responsive electrical signals to the liquid stimulus because of their poor conductivity.

Based on the aforementioned discussion, the liquid sensing mechanism of the hierarchical CB@CNs/NR nanocomposites is proposed in Figure S3. CB@CNs nanohybrid is selectively located in the interstitial space between the NR latex microspheres and formed a 3D hierarchical conductive structure in sample preparation. When the sample is exposed to organic liquids, organic molecules penetrate into the NR matrix and accumulate, followed by swelling of the NR matrix. This causes the disconnection of the 3D hierarchical conductive networks, accompanied with the drastic increase of electric resistance. The sensitivity of this CPC to organic liquid stimuli is severely affected by the 
density of conductive networks, i.e. the contents of $\mathrm{CB} @ \mathrm{CNs}$ nanohybrid. "Brittle" conductive networks are more sensitive to organic liquid stimuli. During the drying process, the hierarchical conductive structure is repaired due to the remove of organic liquids and shrinking of the NR matrix. However, it is difficult for the conductive structures to be reconstructed completely and restored to the original state because of the swelling history and the stubbornly residual solvents in NR matrix ${ }^{[25]}$. Hence, 3D hierarchical conductive structures fabricated with lower filler content show faster response to organic liquid stimuli but lower responsivity as well as poor recover performance, while conductive structures fabricated with higher filler content show slower response but higher responsivity and better recover performance. Thus, tunable liquid sensing performance of this CPC can be achieved, which makes it more promising for the large scale fabrication and application of this CNs modulated hierarchical CPCs.

In addition to rapid response and high responsivity, a good reproducibility is also necessary for a promising sensing material. To evaluate the reproducibility of this sensing material in service, consecutive liquid sensing runs was performed in toluene. As depicted in Figure 5a, the liquid toluene could be reproducibly detected for eleven times with nearly identical sensing behaviors, indicating the excellent reproducibility of this sensing material. Besides, the liquid sensing properties of this $\mathrm{CB} @ \mathrm{CNs} / \mathrm{NR}$ nanocomposite in different organic solvents were investigated. Figure $5 \mathrm{~b}$ shows the responsivity vs. time plots of this CPC in petroleum ether, chloroform, toluene, cyclohexane, n-hexane and xylene, respectively. It is noticed that analyte which has smaller molar volume and lower boiling temperature generally exhibited faster response. This is attributed to that smaller molar volume and lower boiling temperature contribute to the faster penetration of solvent molecules into the NR matrix, thus cause breakage of the $3 \mathrm{D}$ hierarchical conductive networks ${ }^{[34]}$. Due to the different sensing 
response rates in various organic solvents, this $\mathrm{CB} @ \mathrm{CNs} / \mathrm{NR}$ nanocomposite might be utilized to not only detect but also distinguish various organic solvents.

Based on that the 3D hierarchical conductive structure modulated by CNs exhibited good liquid sensing behavior, here, a preliminary study of strain sensing properties of this $\mathrm{CB} @ \mathrm{CNs} / \mathrm{NR}$ nanocomposite for potential strain sensors was conducted. A typical strain sensing behavior of $\mathrm{CB} @ \mathrm{CNs} / \mathrm{NR}$ nanocomposite $(5.0 \mathrm{vol} \% \mathrm{CB})$ subjected to cyclic straining is shown in Figure 6a. It is noticed that a nearly linear change in resistance vs. strain is observed during both loading and unloading process. This linear change in resistance vs. strain demonstrates the potential use of this material as a gauge to measure strains higher than the $5 \%$ limit of conventional metal strain gauges ${ }^{[32]}$. However, a small hysteresis could be seen, and the initial resistance shows a slight increase after the cyclic straining. This sensitivity loss is a common feature of elastomer based strain sensors due to the rearrangement of the conductive fillers and creep of the elastomeric matrix ${ }^{[35-39]}$. Further tests were carried out to investigate the reproducibility of this sensor material. Figure $6 \mathrm{~b}$ gives the responsivity change in ten loading-unloading cycles at a strain amplitude of $20 \%$. After the initial cycle, a repeatable electrical signal was obtained, demonstrating good reproducibility of this 3D hierarchical conductive structure based strain sensor. These results demonstrate the potential application of this CB@CNs/NR nanocomposite in strain sensing fields, such as human motion detection, strain gauge and internal microcrack monitor ${ }^{[40]}$. This is just a preliminary exploration of this material in strain sensors and further work is required to continue this study.

\section{Conclusions}

In summary, we successfully developed a simple and effective approach to a CB-based sensing material with excellent and tunable sensing performance. The fabrication of a 3D hierarchical conductive structure realized the high responsivity (4427), faster response (168 s), high electrical 
conductivity $(0.35 \mathrm{~S} / \mathrm{m})$ and good reproducibility of the CB@CNs/NR nanocomposite containing 3.75 vol\% CB. Furthermore, the organic liquid sensing behaviors of this CPC can be tuned by manipulating the 3D hierarchical conductive networks using different $\mathrm{CB} @ \mathrm{CNs}$ loadings. Finally, the potential application of this material as strain sensor was investigated. This work opens up new opportunities for the facile, low cost and environmentally friendly production of CB based flexible sensing materials with high performance.

\section{Notes}

The authors declare no competing financial interest.

\section{Acknowledgements}

The authors thank the National Science Foundation of China (51203105 and 51473100) and State Key Laboratory of Polymer Materials Engineering (Grant No. sklpme2015-3-04) for financial support.

\section{Supporting Information}

Optical microscope image of pure CB; digital pictures of CB and CB @ CNs suspensions; TEM image of NR latex microspheres; TEM images of CNs; SEM image of the extracted CB@CNs nanohybrid skeleton from CB@CNs/NR nanocomposite. These date can be found in Supporting Information.

\section{Reference}

[1] Piana F.; Pionteck J. Effect of the melt processing conditions on the conductive paths formation in thermoplastic polyurethane/expanded graphite (TPU/EG) composites. Compos. Sci. Technol. 2013, 80, 39-46.

[2] Bora C.; Bharali P.; Baglari S.; Dolui S. K.; Konwar B. K. Strong and conductive reduced graphene oxide/polyester resin composite films with improved mechanical strength, thermal stability and its antibacterial activity. Compos. Sci. Technol. 2013, 87, 1-7. 
[3] Paszkiewicz S.; Szymczyk A.; Sui X. M.; Wagner H. D.; Linares A.; Ezquerra T. A.; Rosłaniec Z. Synergetic effect of single-walled carbon nanotubes (SWCNT) and graphene nanoplatelets (GNP) in electrically conductive PTTblock-PTMO hybrid nanocomposites prepared by in situ polymerization. Compos. Sci. Technol. 2015, 118, $72-77$.

[4] Ventura I. A.; Zhou J.; Lubineau G. Drastic modification of the piezoresistive behavior of polymer nanocomposites by using conductive polymer coatings. Compos. Sci. Technol. 2015, 117, 342-350.

[5] Xu R.; Lu Y.; Jiang C.; Chen J.; MaoP.; Gao G.; Zhang L.; Wu S. Facile Fabrication of Three-Dimensional Graphene Foam/Poly(dimethylsiloxane) Composites and Their Potential Application as Strain Sensor. ACS Appl. Mater. Interfaces 2014, 6, 13455-13460.

[6] Lee J.; Kwon H.; Seo J.; Shin S.; Koo J. H.; Pang C.; Son S.; Kim J. H.; Jang Y. H.; Kim D. E.; Lee T. Conductive Fiber-Based Ultrasensitive Textile Pressure Sensor for Wearable Electronics. Adv. Mater. 2015, 27, 2433-2439.

[7] Carrillo A.; Martín-Domínguez I. R.; Rosas A.; Márquez A. Numerical method to evaluate the influence of organic solvent absorption on the conductivity of polymeric composites. Polymer, 2002, 43, 6307-6313.

[8] Mohiuddin M.; Hoa S.V. Temperature dependent electrical conductivity of CNT-PEEK composites. Compos. Sci. Technol. 2011, 72, 21-27.

[9] Wan Y.; Xiong C.; Yu J.; Wen D. Effect of processing parameters on electrical resistivity and thermo-sensitive properties of carbon-black/styrene-butadiene-rubber composite membranes. Compos. Sci. Technol. 2005, 65, 17691779.

[10] Wu C.; Huang X.; Wang G.; Lv L.; Chen G.; Li G.; Jiang P. Highly Conductive Nanocomposites with ThreeDimensional, Compactly Interconnected Graphene Networks via a Self-Assembly Process. Adv. Funct. Mater. 2013, 23, 506-513.

[11] Yan D. X.; Pang H.; Li B.; Vajtai R.; Xu L.; Ren P. G.; Wang J. H.; Li Z. Structured Reduced Graphene Oxide/Polymer Composites for Ultra-Efficient Electromagnetic Interference Shielding. M. Adv. Funct. Mater. 2015, $25,559-566$. 
[12] Potts J. R.; Shankar O.; Du L.; Ruoff R. S. Processing-Morphology-Property Relationships and Composite Theory Analysis of Reduced Graphene Oxide/Natural Rubber Nanocomposites. Macromolecules 2012, 45, 60456055.

[13] Zhan Y.; Lavorgna M.; Buonocore G.; Xia H. Enhancing Electrical Conductivity of Rubber Composites by Constructing Interconnected Network of Self-assembled Graphene with Latex Mixing. J. Mater. Chem. 2012, 22, 10464-10468.

[14] Yang L.; Wang Z.; Ji Y.; Wang J.; Xue G. Highly Ordered 3D Graphene-Based Polymer Composite Materials Fabricated by "Particle-Constructing" Method and Their Outstanding Conductivity. Macromolecules 2014, 47, 17491756.

[15] Tang C.; Long G.; Hu X.; Wong K.; Lau W.; Fan M.; Mei J.; Xu T.; Wang B.; Hui D. Conductive Polymer Nanocomposites with Hierarchical Multi-scale Structuresvia Self-assembly of Carbon-Nanotubes on Graphene on Polymer-Microspheres. Nanoscale 2014, 6, 7877-7888.

[16] George N.; Chandra C.S. J.; Mathiazhagan A.; Joseph R. High performance natural rubber composites with conductive segregated network of multiwalled carbon nanotubes. Compos. Sci. Technol. 2015, 116, 33-40

[17] Noël A.; Faucheu J.; Rieu M.; Viricelle J.P.; Bourgeat-Lami E. Tunable architecture for flexible and highly conductive graphene-polymer composites. Compos. Sci. Technol. 2014, 95, 82-88.

[18] Yoonessi M.; Gaier J. R. Highly Conductive Multifunctional Graphene Polycarbonate Nanocomposites. ACS Nano 2010, 4, 7211-7220.

[19] Wu, X. D.; Lu, C. L.; Xu, H. Y.; Zhang, X. X.; Zhou, Z. H. Biotemplate Synthesis of Polyaniline@Cellulose Nanowhiskers/Natural Rubber Nanocomposites with 3D Hierarchical Multiscale Structure and Improved Electrical Conductivity. ACS Appl. Mater. Interfaces 2014, 6, 21078-21085.

[20] Zhang X.; Wu X.; Lu C.; Zhou Z. Dialysis-Free and in Situ Doping Synthesis of Polypyrrole@Cellulose Nanowhiskers Nanohybrid for Preparation of Conductive Nanocomposites with Enhanced Properties. ACS Sustainable Chem. Eng. 2015, 3, 675-682. 
[21] Wu X.; Lu C.; Zhang X.; Zhou Z. Conductive natural rubber/carbon black nanocomposites via cellulose nanowhisker templated assembly: tailored hierarchical structure leading to synergistic property enhancements. $\boldsymbol{J}$. Mater. Chem. A 2015, 3, 13317-13323.

[22] Villmow T.; Pegel S.; Pötschke P.; Heinrich G. Polymer/carbon nanotube composites for liquid sensing: Model for electrical response characteristics. Polymer, 2011, 52, 2276-2285.

[23] Wu X.; Lu C.; Zhou Z.; Yuan G.; Xiong R.; Zhang X. Green synthesis and formation mechanism of cellulose nanocrystal-supported gold nanoparticles with enhanced catalytic performance. Environ. Sci.: Nano, 2014, 1, 71-79.

[24] Pang H.; Bao Y.; Xu L.; Yan D. X.; Zhang W. Q.; Wang J. H.; Li Z. M. Double-segregated carbon nanotubepolymer conductive composites as candidates for liquid sensing materials. J. Mater. Chem. A, 2013, 1, 4177-4181.

[25] Pang H.; Piao Y. Y.; Xu L., Bao Y.; Cui C. H.; Fu Q.; Li Z. M. Tunable Liquid Sensing Performance of Conducting Carbon Nanotube-Polyethylene Composites with a Porous Segregated Structure. RSC Adv. 2013, 3, 19802-19806.

[26] Villmow T.; John A.; Pötschke P.; Heinrich G. Polymer/Carbon Nanotube Composites for Liquid Sensing: Selectivity against Different Solvents. Polymer 2012, 53, 2908-2918.

[27] Pötschke P.; Kobashi K.; Villmow T.; Andres T.; Paiva M. C.; Covas J. A. Liquid Sensing Properties of Melt Processed Polypropylene/Poly(E-caprolactone) Blends Containing Multiwalled Carbon Nanotubes. Compos. Sci. Technol. 2011, 71, 1451-1460.

[28] Ravikiran Y. T.; Kotresh S.; Vijayakumari S. C.; Thomas S. Liquid Petroleum Gas Sensing Performance of Polyaniline-Carboxymethyl Cellulose Composite at Room Temperature. Curr. Appl. Phys. 2014, 14, 960-964.

[29] Pötschke P.; Andres T.; Villmow T.; Pegel S.; Brünig H.; Kobashi K.; Fischer D.; Häussler L. Liquid Sensing Properties of Fibres Prepared by Melt Pinning from Poly(lactic acid) Containing Multi-walled Carbon Nanotubes Compos. Sci. Technol. 2010, 70, 343-349. 
[30] Segal E.; Tchoudakov R.; Mironi-Harpaz I.; Narkis M.; Siegmann A. Chemical Sensing Materials Based on Electrically-conductive Immiscible Polymer Blends. Polym. Int. 2005, 54, 1065-1075.

[31] Tsubokawa N.; Tsuchida M.; Chen J.; Nakazawa Y. A Novel Contamination Sensor in Solution: the Response of Electric Resistance of a Composite Based on Crystalline Polymer-grafted Carbon Black. Sens. Actuators B 2001, 79, 92-97.

[32] Narkis M.; Srivastava S.; Tchoudakov R.; Breuer O. Sensors for Liquids Based on Conductive Immiscible Polymer Blends. Synthetic Met. 2000, 113, 29-34.

[33] Segal E.; Tchoudakov R.; Narkis M.; Siegmann A. Sensing of Liquids by Electrically Conductive Immiscible Polypropylene/Thermoplastic Polyurethane Blends Containing Carbon Black. J. Polym. Sci. Poly. Phys. 2003, 41, $1428-1440$.

[34] Dai K.; Zhao S.; Zhai W.; Zheng G.; Liu C.; Chen J.; Shen C. Tuning of Liquid Sensing Performance of Conductive Carbon Black (CB)/Polypropylene (PP) Composite Utilizing a Segregated Structure. Compos. Part A 2013, 55, 11-18.

[35] Yamada T.; Hayamizu Y.; Yamamoto Y.; Yomogida Y.; Izadi-Najafabadi A.; Futaba D. N.; Hata K. A Stretchable Carbon Nanotube Strain Sensor for Human-Motion Detection. Nat. Nanotechnol. 2011, 6, 296-301.

[36] Wang S. M.; Zhang X. X.; Wu X. D.; Lu C. H. Tailoring percolating conductive networks of natural rubber composites for flexible strain sensors via a cellulose nanocrystal templated assembly. Soft matter DOI: $10.1039 / \mathrm{c} 5 \mathrm{sm} 01958 \mathrm{c}$.

[37] Martinez F.; Obieta G.; Uribe I.; Sikora T.; Ochoteco E. Polymer-Based Self-Standing Flexible Strain Sensor. J. Sensors 2010, 659571.

[38] Zavickis J.; Knite M.; Podins G.; Linarts A.; Orlovs R. Polyisoprene-Nanostructured Carbon Composite-A Soft Alternative for Pressure Sensor Application. Sens. Actuators A 2011, 171, 38-42. 
[39] Levin Z. S.; Robert C.; Feller J. F.; Castro M.; Grunlan J. C. Flexible Latex-Polyaniline Segregated Network Composite Coating Capable of Measuring Large Strain on Epoxy. Smart Mater. Struct. 2013, 22, 015008.

[40] Zhang J.; Liu J.; Zhuang R.; Mäder E.; Heinrich G.; Gao S. Single MWNT-Glass Fiber as Strain Sensor and Switch. Adv. Mater. 2011, 23, 3392-3397.

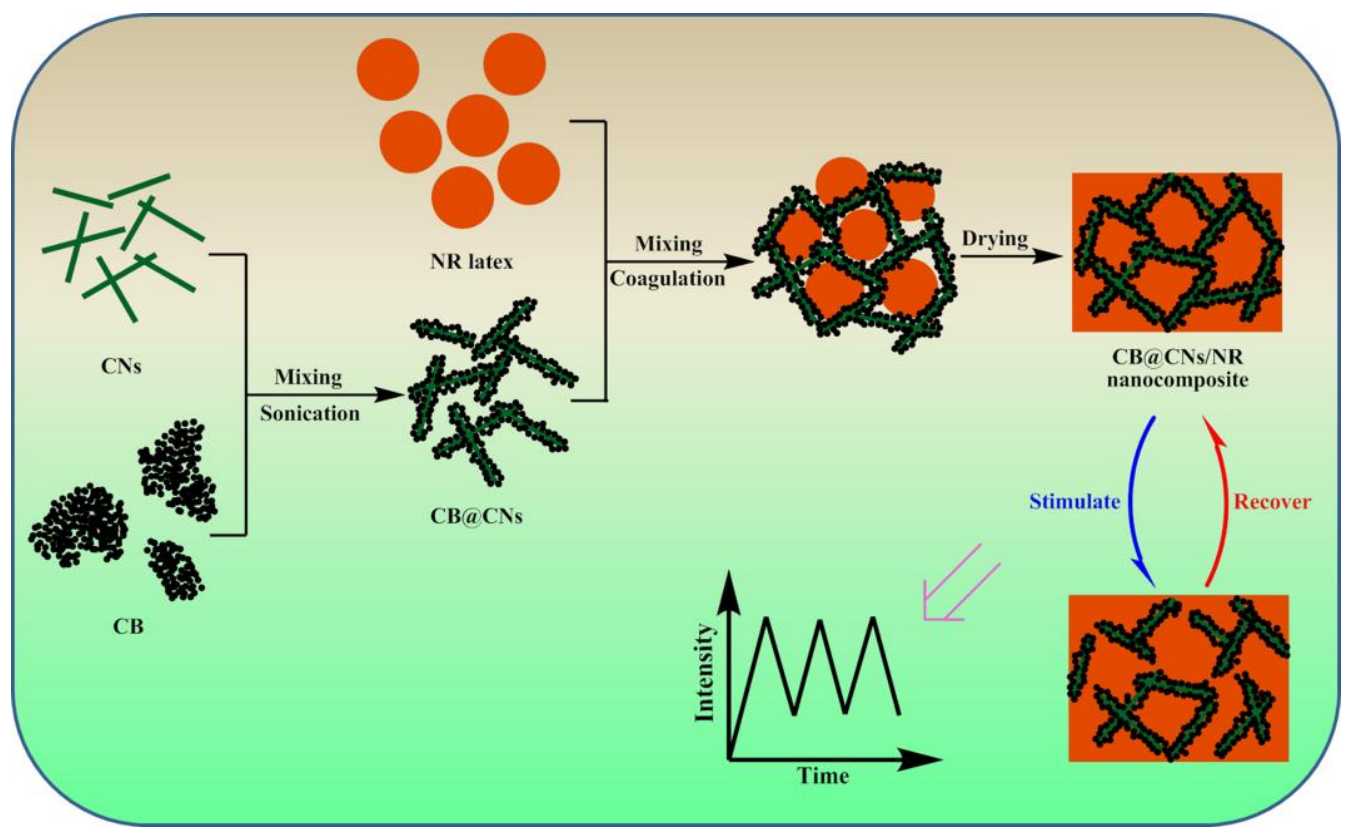

Figure 1. Schematic illustration for $\mathrm{CNs}$ modulated fabrication of $\mathrm{CB}$ based 3D hierarchical conductive structure in NR matrix for sensing application 

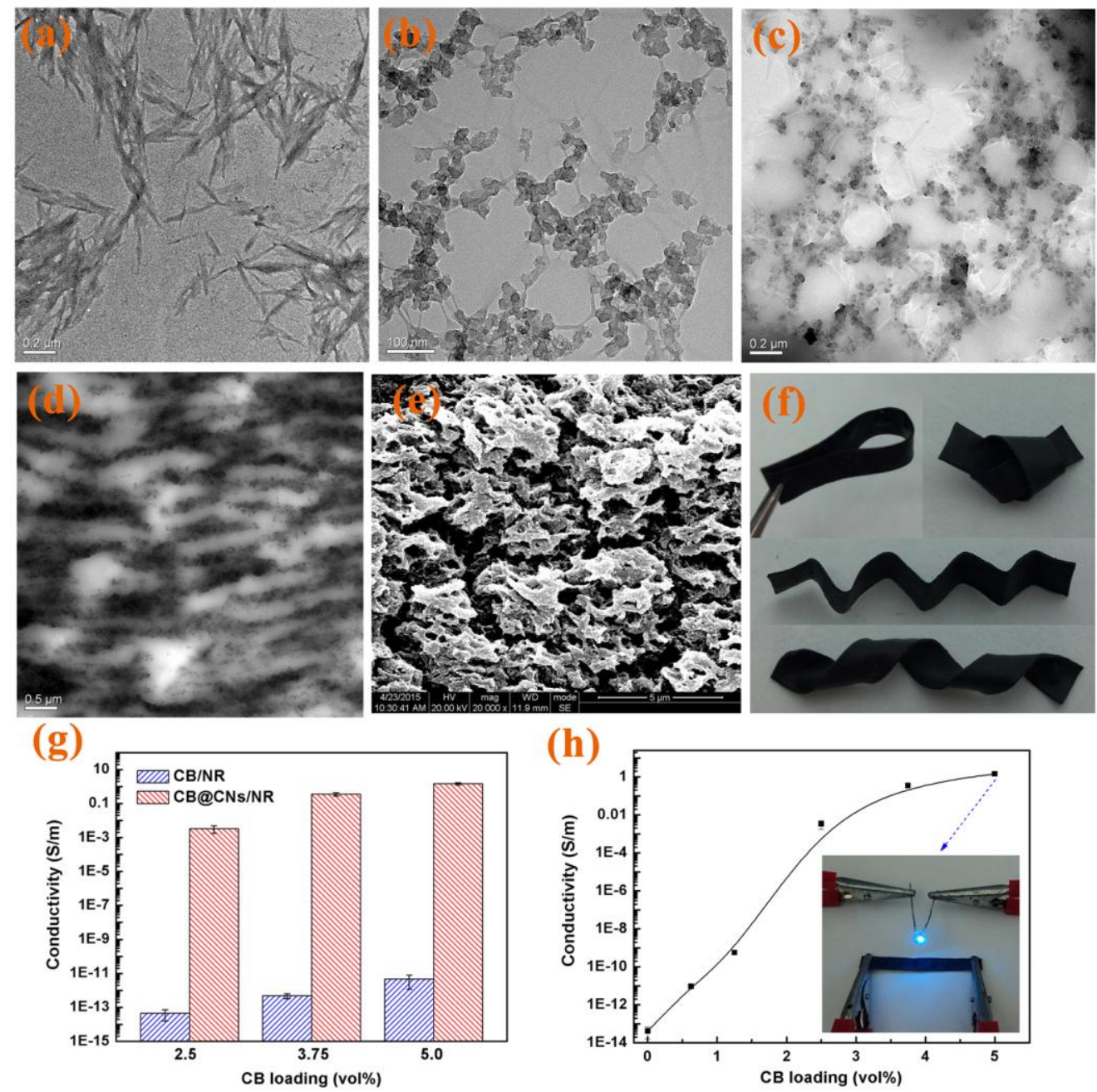

Figure 2. TEM images of CNs (a, scale bar: 200 nm); TEM images of CB@CNs nanohybrid (b, scale bar: 100 nm); TEM images of CB @CNs nanohybrid and NR latex mixture (c, scale bar: 200 nm); TEM images of CB@CNs/NR nanocomposite prepared via latex assembly technique (d, scale bars: $500 \mathrm{~nm})$; SEM image of the extracted CB@CNs nanohybrid skeleton from CB@CNs/NR nanocomposite (e scale bars: $5 \mu \mathrm{m}$ ); digital picture of flexible $\mathrm{CB} @ \mathrm{CNs} / \mathrm{NR}$ nanocomposite samples (f); electrical conductivity comparison of $\mathrm{CB} @ \mathrm{CNs} / \mathrm{NR}$ and $\mathrm{CB} / \mathrm{NR}$ nanocomposites with different volume fraction of CB (g); electrical conductivity of CB@CNs/NR nanocomposites as a function of CB content (vol\%) (h), the inset shows CB@CNs/NR nanocomposite light up an LED device by $9 \mathrm{~V}$ voltage. 


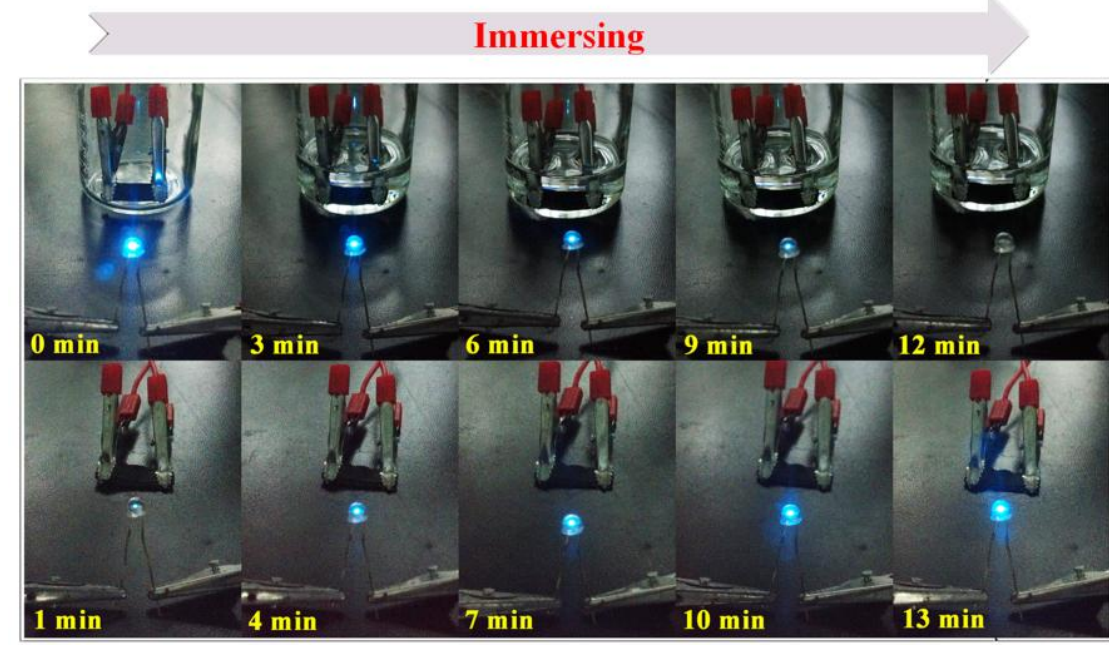

Drying

Figure 3. Photographs of illumination changes during $\mathrm{CB} @ \mathrm{CNs} / \mathrm{NR}$ sample (5 vol\% $\mathrm{CB})$ immersing into toluene and drying by hot-air
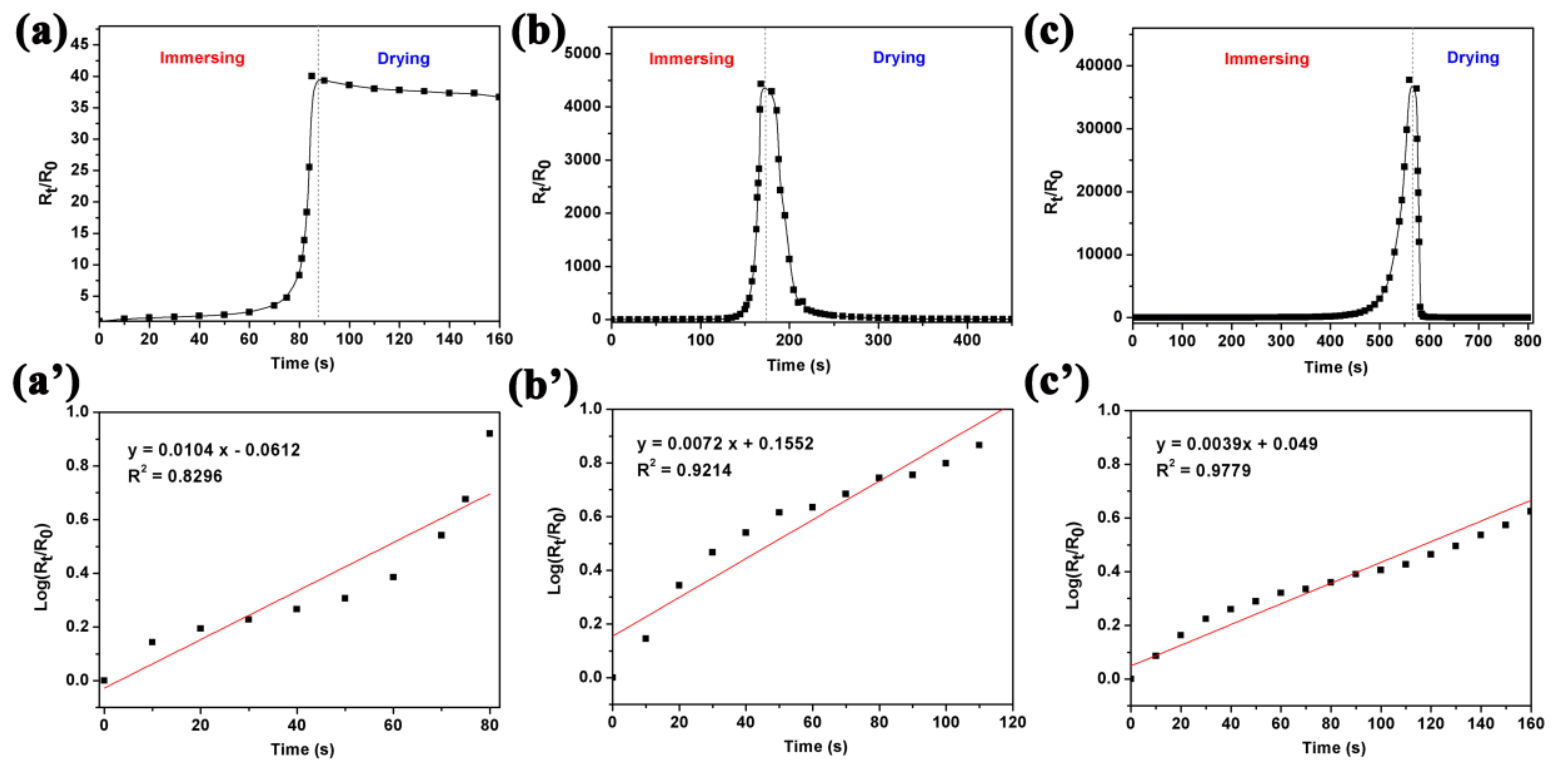

Figure 4. Responsivity vs. time and plots of $\log \left(\mathrm{R}_{\mathrm{t}} / \mathrm{R}_{0}\right)$ vs. time during immersing-drying process for the CB@CNs/NR nanocomposites filled with different CB loading: 2.5 vol\% (a, a'), 3.75 vol\% (b, b'), and $5.0 \mathrm{vol} \%$ (c, c') 
(a)

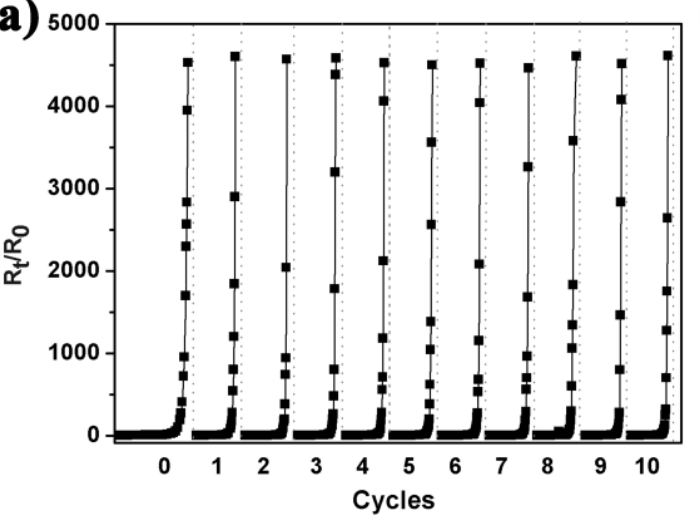

(b)

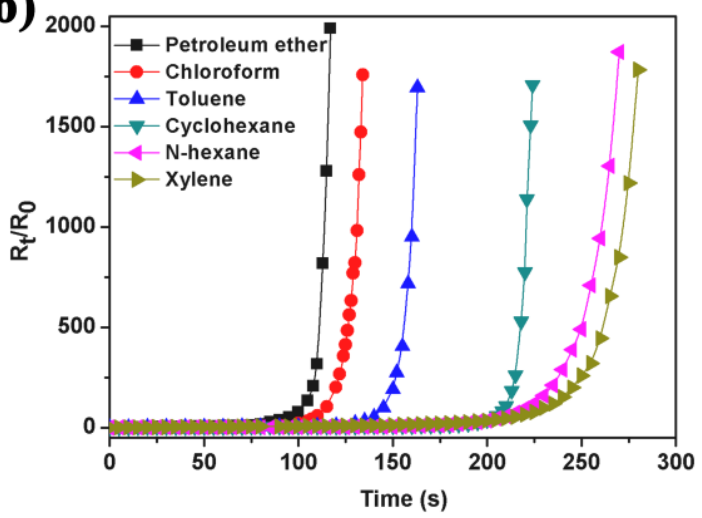

Figure 5. Responsivity change of the CB@CNs/NR nanocomposite (3.75 vol\% CB): (a) during eleven immersing runs in toluene and (b) exposed to different organic liquids
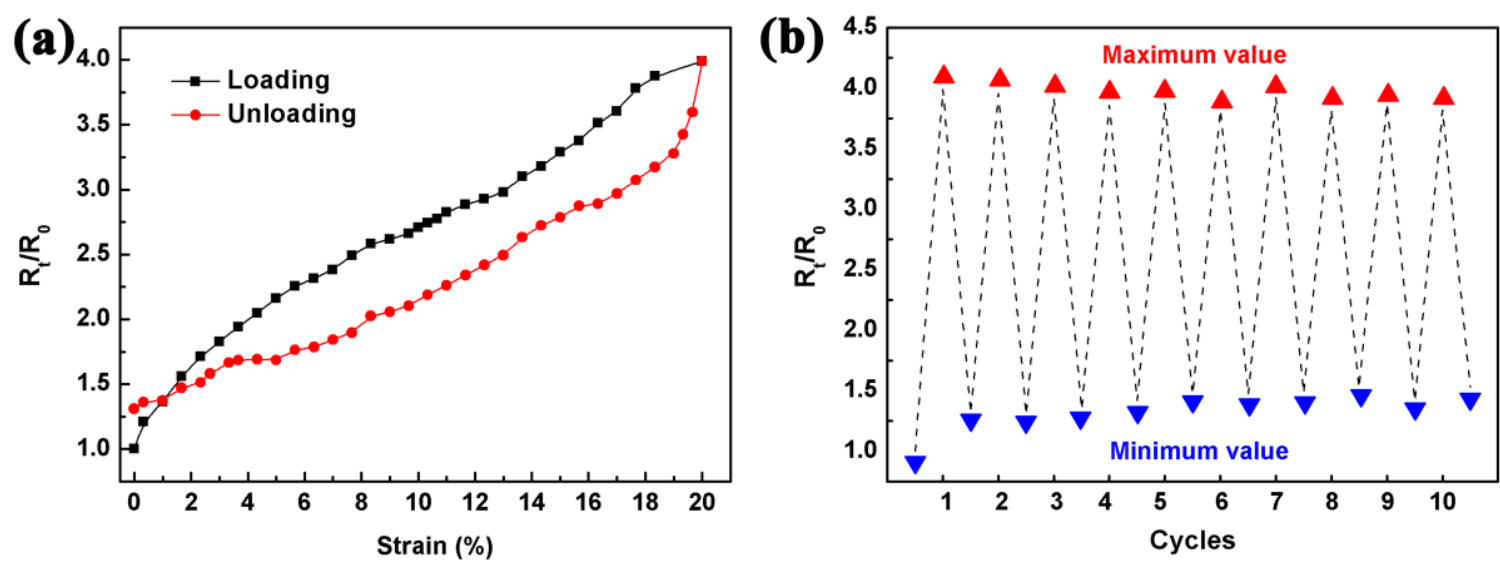

Figure 6. Responsivity vs. strain of $\mathrm{CB} @ \mathrm{CNs} / \mathrm{NR}$ nanocomposite $(5.0$ vol\% $\mathrm{CB})$ during a loadingunloading cycle (a) and responsivity change in ten loading-unloading cycles (b) at a strain amplitude of $20 \%$ 
Table 1. Comparison of liquid sensing performance of various CPCs

\begin{tabular}{llll}
\hline Samples & Response time(s) & Responsivity & References \\
\hline PC/CNT & $1000-12000$ & $1.7-2000$ & 26 \\
PP/PCL/MWNT & 600 & $0-4$ & 27 \\
PANI/CMC & $200-400$ & $2-34$ & 28 \\
PLA/MWNT & $120-600$ & $0-1.9$ & 29 \\
PP/TPU/CB & 120 & 3.4 & 30 \\
PPCL/PEG-g-CB & 600 & $4-50$ & 31 \\
HIPS/EVA/CB & 900 & $1-900$ & 32 \\
PP/TPU/CB & 180 & $3-25$ & 33 \\
CB/NR (2.5\% - 5\% CB) & - & -37743 & this work \\
CB/CNs/NR (2.5\% CB) & 85 & 38.6 & this work \\
CB/CNs/NR (3.75\% CB) & 168 & 4427 & this work \\
CB/CNs/NR (5.0\% CB) & 560 & & \\
\end{tabular}

Note: polycarbonate-PC; carbon nanotubes-CNT; polypropylene-PP; thermoplastic polyurethane-TPU; poly(ecaprolactone)-PCL; multiwalled carbon nanotubes-MWNT; polyaniline-PANI; carboxymethyl cellulose-CMC; poly(lactic acid)-PLA; poly(ethylene glycol)-PEG; high impact polystyrene-HIPS; ethylene vinyl acetate copolymerEVA. 